\title{
Auf dem Weg zur "Großmacht": Die Presse im 19. Jahrhundert
}

I.

Nicht selten ist heute von der "Macht" der Massenmedien die Rede. DaB die Massenmedien Macht besitzen, wird zwar auch bestritten, zumal von denjenigen, die selbst über diese Macht verfügen, nämlich den Journalisten. Und die etwas metaphorisch klingende Formulierung läßt zunächst auch offen, was hier mit „Macht" gemeint ist. Ob dieser herkömmlich für die politische Analyse zentrale Begriff überhaupt noch adäquat ist, wird zudem seit einiger Zeit in Zweifel gezogen. ${ }^{1}$ Infolgedessen sprechen manche Autoren neuerdings auch eher von "Einfluß". Ohne auf diesen theoretisch vielleicht bedeutsamen Unterschied im Sprachgebrauch hier weiter einzugehen, läßt sich doch kaum übersehen, daß es Indikatoren für das Machtpotential der Massenmedien gibt. Dazu gehört vor allem die Chance, Information und Kritik massenhaft zu verbreiten, und zwar einseitig (vom Sender zum Empfänger) und öffentlich (so dab es jeder erfahren kann).

Die Erkenntnis oder zumindest das Gefühl, die Massenmedien besäßen Macht, ist jedoch nicht neu. Historische Belege, daß man sie schon früher wahrnahm, lassen sich leicht erbringen. So ist die Bemerkung des österreichischen Staatskanzlers Metternich überliefert: „Die Presse ist eine Gewalt. Gewalten müssen, sollen sie nicht gefährlich sein, stets geregelt werden." ${ }^{2}$ Welche ${ }_{n}$ Regelung" damit gemeint war, wissen wir: Es waren die Karlsbader Beschlüsse von 1819, die in Deutschland (wieder) eine Epoche strenger Zensur herbeiführen sollten. Als weiterer Gewährsmann für diese Erkenntnis wird immer wieder Napoleon genannt, ${ }^{3}$ der diese vermutlich der Französischen Revolution verdankte und daraus ähnliche Konsequenzen zog wie später Metternich. Sinngemäße Wendungen lassen sich sogar noch früher finden, ${ }^{4}$ ja unausgesprochen dürfte die Annahme

1 Vgl. Ronneberger 1983.

2 So Metternich an Bülow am 24.09.1842, zit. nach Hoefer 1983, S. 49.

3 Geradezu zum geflügelten Wort wurde der von Napoleon überlieferte Ausspruch: „Que quatre gazettes hostiles faisaient plus de mal, que cent milles hommes en plate campagne."

"Hinweise darauf finden sich z.B. in den „Federalists", jener Sammlung von Abhandlungen, die von den Gründern der Vereinigten Staaten über Verfassungsfragen $1787 / 88$ geschrieben wurden. Vgl. Wilson 1942, Cooke 1961. 
einer "Macht" der publizistischen Medien hinter allen Versuchen stehen, diese einer Kontrolle zu unterwerfen.

So sehr sich also Vorstufen zeigen, die Einsicht von der Macht der Massenmedien kam erst im 19. Jahrhundert voll zum Durchbruch. Und dies hatte, wie im folgenden dargestellt werden soll, seine Gründe. Dabei fand insofern noch eine gewisse Steigerung statt, als damals die Rede von der Presse als einer "Großmacht" aufkam. Dies geschah in einer ganzen Reihe von Schriften, die im letzten Drittel des 19. Jahrhunderts erschienen sind. „Die Großmacht der Presse" - so lautet der Titel schon einer der ersten von ihnen, die 1866 publiziert wurde. „Wer immer, der auch nur flüchtig die Lage der Dinge in der Gegenwart betrachtet", so führt der Verfasser darin aus, ,kann es sich verhehlen, daß unsere Zeit zum großen Theile gerade durch die Presse das geworden ist, was sie ist. Wer vermag zu leugnen, daß bis zur Stunde die Presse als ein Hauptmittel benützt und ausgebeutet wird, um Throne zu stürzen, um den Altar zu zertrümmern, um Königreiche zu erobern, um Völker zu unterjochen und zu gängeln, indem man sie verwirrt und irre leitet." ${ }^{5}$ Der Autor vergleicht - in militärischer Analogie - die Presse ausdrücklich mit einer Armee und resümiert: „Die Tagespresse ist eine GroBmacht, die sich nicht ignoriren läßt und deren Eingreifen in die Geschicke der Völker um so gewaltiger erscheint, je weniger sie wählerisch ist in ihren Mitteln."

Verfaßt hatte diese Schrift der Speyerer Domkapitular Wilhelm Molitor, wobei dies (mit) erklären mag, was manchem vielleicht als apokalyptische Überzeichnung erscheint. Molitor löste damit indessen eine breite Debatte aus. ${ }^{7}$ Auch wenn das Argument von der Macht der Presse unverkennbar vor allem auf konservativer Seite Zuspruch fand und sich mit dort vorhandenen kulturkritischen Ressentiments verband, so läßt es sich doch nicht darauf reduzieren und damit gar erledigen. Nicht nur die Schriften, die unmittelbar an Molitor anschlossen, nahmen die Formel auf, wie z.B. Joseph Lukas, in dessen Replik es ein Jahr später lapidar hieß: „Die Presse ist eine gewaltige Macht. Dieser Satz bedarf heute keines Beweises mehr, um geglaubt zu werden." (1867, S. 1) Vielmehr wurde dieser Gedanke auch von anderen propagiert. Heinrich Wuttke, einer der schärfsten Kritiker der Presseentwicklung im 19. Jahrhundert, notierte 1875: „Die beherrschende Macht der Tagespresse über den Sinn der Men-

${ }^{5}$ Molitor 1866, S. 6 f.

${ }^{6}$ Molitor 1866, S. 9.

7 Vgl. Lukas 1867, Hammer 1868, Zöckler 1870. 
schen stellt man sich ohnehin gewöhnlich zu gering vor"; ${ }^{8}$ und um diese Vorstellung zu korrigieren, führte er eine ganze Reihe von Beispielen an. Übrigens folgten Schriften wie die hier zitierten auch später noch, zumal nach der Jahrhundertwende. „Ist heute die Presse eine Macht?", fragte 1911 z.B. der Tübinger Redakteur Carl Erler, und er antwortete ebenfalls: „Diese Frage wird und kann kein Mensch verneinen."

\section{II.}

Von den zuvor angeführten Zeugnissen ausgehend, möchte ich im folgenden $\mathrm{zu}$ beschreiben suchen, inwiefern die Presse im 19. Jahrhundert zu einer "Großmacht" wurde bzw. warum sich den Zeitgenossen damals ein solcher Eindruck aufdrängte. Die ersten Druckwerke, welche die Merkmale der Zeitung in vollem Umfang aufweisen (Aktualität, Periodizität, Universalität, Publizität), waren in Deutschland zwar schon im frühen 17. Jahrhundert erschienen. Und dieses Medium entwickelte sich hier rascher und vielfältiger als in allen anderen Ländern Europas, was vor allem mit dem deutschen Territorialismus zu tun hatte. Doch so sehr sich die Zahl der Zeitungen schon vermehrte und eine inhaltliche Erweiterung einsetzte - bis zum Ende des 18. Jahrhunderts veränderte sich das Medium nur wenig.

Der grundlegende Wandel, ja der Durchbruch zur modernen Presse vollzog sich hingegen erst im 19. Jahrhundert. Das wird deutlich, wenn man die Situation am Ende des 18. mit der am Ende des 19. oder am Beginn des 20. Jahrhunderts vergleicht. Zum Beleg dazu führe ich im folgenden Daten aus verschiedenen Quellen an. Einerseits handelt es sich um quantitative Erhebungen aus der damaligen Zeit selbst oder solche, die im frühen 20. Jahrhundert vorgenommen wurden. ${ }^{10}$ Sie sind der ersten Phase einer Pressestatistik in Deutschland zu verdanken, die sich vor allem auf Postzeitungslisten und Zeitungskataloge stützte. Wenngleich die seinerzeit ermitteiten Zahlen eine Grundlage hatten, die man für das 18. Jahrhundert noch nicht besitzt, so standen sie doch auf unsicherem Boden. Dies nicht nur, weil diese Quellen unterschiedlich vollständig sind, sondern auch, weil die Erhebungseinheit "Zeitung” variiert oder variieren kann. Aber selbst wenn man einräumt, es nur mit Annäherungswerten

8 Wuttke 1875, S. 194.

9 Erler 1911. Allerdings relativiert Erler diese Aussage im folgenden mit der Bemerkung, „daß diese Macht und dieser Einfluß doch nur recht beschränkt ist" (S. 26).

10 Vgl. Schacht 1898, La statistique des journaux 1908 (hier insbesondere S. 178, 179), Stoklossa 1913, Muser 1918. 
zu tun zu haben, bleiben diese wichtig genug. In zweiter Linie greife ich auf eine von mir selbst durchgeführte jüngere Untersuchung zurück. ${ }^{11}$ Das Beispiel des "Hamburgischen Unpartheyischen Correspondenten", um den es dabei vor allem geht, mag auch nicht repräsentativ sein für die gesamte deutsche Tagespresse. Da die Studie aber bisher vereinzelt ist, gibt es keine Alternative, wenn man bestimmte Dinge quantitativ belegen will.

Nach erst in neuerer Zeit gemachten Erhebungen bestanden am Ende des 17. Jahrhunderts im deutschsprachigen Raum bereits etwa 70 Zeitungen. ${ }^{12}$ Am Ende des 18. Jahrhunderts waren es über 200. Das ergab eine nicht unbeträchtliche Zeitungsdichte. Doch im 19. Jahrhundert sollte erst recht eine enorme Expansion im Pressewesen einsetzen. So konnte Hjalmar Schacht am Ende dieses Jahrhunderts (1897) in Deutschland insgesamt 3405 Zeitungen zählen. ${ }^{13}$ Auch wenn die Erhebungseinheit nicht kongruent sein mag, die nur als Kopfblätter erscheinenden Zeitungsausgaben einzeln gezählt sein dürften und man die typologische Differenzierung des Mediums in Rechnung stellen muB, so ist die Vervielfachung des Zeitungsangebots doch unübersehbar. Erschienen am Ende des 18. Jahrhunderts Zeitungen in etwa 150 Orten, so registrierte Schacht im Jahre 18971884 Verlagsorte. Zahlreiche Städte und Gemeinden, die am Ende des 18. Jahrhunderts noch keine Zeitung besaßen, verfügten ein Jahrhundert später über eine solche, in anderen waren weitere Titel hinzugekommen. Im ganzen war das Netz der Zeitungen enger geworden, die Dispersion des Mediums hatte sich bis in regionale und lokale Kleinräume - „bis in den entferntesten Winkel des Landes"14 - fortgesetzt.

Die hier durch „Randwerte” belegte Expansion des Pressewesens im 19. Jahrhundert hat sich naturgemäß nicht kontinuierlich vollzogen. Vielmehr kam es dazu in mehreren Schüben, die mit bestimmten politischen und wirtschaftlichen Ursachen zusammenhingen. Vor 1848 lag die Zahl der Zeitungen (einschließlich Intelligenzblätter) offenbar um die $1000{ }^{15}$ Erst infolge der März-Revolution und der durch sie proklamierten Pressefreiheit kam es zu einer größeren Gründungswelle bei den politischen

11 Vgl. Wilke 1984.

12 Vgl. Bogel/Blühm 1971, 1985.

13 Schacht 1898, S. 508.

14 Zöckler 1870, S. 20.

15 Vgl. von Reden 1848. 
Zeitungen. Diese kam jedoch wegen neuerlicher restriktiver Bestimmungen schon alsbald zum Erliegen. Eine weitere Welle der Etablierung neuer Zeitungen folgte in den siebziger Jahren, eine dritte läßt sich in den achtziger Jahren beobachten. Werden in den vorliegenden Statistiken für 18621300 Zeitungen genannt, so für 18812437 und für 18913005 . Die Dynamik der Expansion lag demnach in der zweiten Hälfte, ja in den späteren Jahrzehnten des 19 . Jahrhunderts.

Eine zweite Ebene, auf der es zu einer Expansion im Pressewesen kam, bilden die Auflagen, d.h. die Anzahl der gedruckten Exemplare. Aufgrund neuerer Berechnungen wird die Gesamtauflage der deutschen $\mathrm{Ta}$ gespresse am Ende des 18. Jahrhunderts auf 300000 Exemplare (pro Erscheinungsintervall) geschätzt. ${ }^{16}$ Dabei wird für die größeren Blätter eine Durchschnittsauflage von 4000 Exemplaren zugrunde gelegt. Schon hier hat man gemeint, von "Massenpresse" sprechen zu können, zumal wenn die im 18. Jahrhundert noch beträchtliche $Z$ ahl von durchschnittlich zehn Lesern pro Exemplar angenommen wird (was auf eine Zeitungsleserschaft von ca. 3 Millionen bei weniger als 20 Millionen Einwohnern schließen läßt). Schacht errechnete 1897 für die von ihm gezählten Zeitungen eine Gesamtauflage von 12,2 Millionen Exemplaren. ${ }^{17}$ Dies entspräche einer Steigerung um das Vierzigfache binnen eines Jahrhunderts. Als Durchschnittsauflage pro Zeitung im Reichsgebiet ergeben sich dabei 3660 Exemplare. Das ist im Vergleich zu heute immer noch wenig. Aber schon damals standen neben einer großen Anzahl kleinauflagiger Blätter bereits einige mit hohen Auflagen, so der 1893 von August Scherl gegründete "Berliner Lokalanzeiger”, der in einer Auflage von 150000 Exemplaren erschien. "GroB" waren jedoch Zeitungen schon, wenn ihre Auflage mehr als 20000 betrug. Die Reichweite der Tagespresse am Ende des 19. Jahrhunderts wird im übrigen dadurch bestimmt, daß eine geringere Zahl von Lesern als früher sich ein Exemplar teilt. Der Zeitungsbezug und die Lektüre, im 18. Jahrhundert noch großenteils in kollektiven Formen gepflegt, hatten sich inzwischen zu individualisieren begonnen. ${ }^{18}$ Bei einer Bevölkerung von über 50 Millionen um 1895 dürfte zumindest weit mehr als die Hälfte Zeitung gelesen haben.

16 Vgl. Welke 1977.

17 Schacht 1898, S. 514. Diese Zahl dürfte, wie Schacht selbst einräumt, etwas höher sein, da er bei den Organen, für die ihm keine Auflagenzahlen vorlagen, Schä̀tzwerte annahm. Als Zusammenstellung von Einzelauflagen vgl. Heenemann 1929.

18 Allerdings gab es den Zeitungsbezug in kollektiven Formen auch noch im 19. Jahrhundert. Vgl. als Beispiel Wilke 1985. 
Von Expansion kann noch in anderweitiger Hinsicht die Rede sein. Waren die ersten Zeitungen, die wir als solche gelten lassen, noch im Wochenrhythmus erschienen (bedingt durch die wöchentlichen Postkurse), so kam es erst nach und nach zu kürzeren Erscheinungsintervallen. Zwar wurde schon 1650 in Leipzig die erste Zeitung täglich herausgegeben ( Einkommende Zeitungen”), doch blieb das eine Ausnahme. Mehr und mehr hatte sich bis zum Ende des 17. Jahrhunderts ein wöchentlich zweimaliges, bis zum Ende des 18. Jahrhunderts ein wöchentlich dreimaliges Erscheinen durchgesetzt. Noch 1897 erschienen nach den Daten von Hjalmar Schacht 30 Prozent der Zeitungen im Deutschen Reich dreimal in der Woche, 20 Prozent zweimal und 11 Prozent einmal. ${ }^{19}$ Doch kam jetzt immerhin schon jede dritte Zeitung (werk-)täglich oder sogar häufiger (mehrmals täglich) heraus. Letzteres war vor allem bei Zeitungen in Berlin oder anderen großen Städten der Fall. Dabei ist der Erscheinungsrhythmus ein entscheidender Indikator für die Dichte, in der das Publikum mit den Botschaften der Zeitung konfrontiert wird.

Bemerkenswert ist vor allem, in welchem Maße der Zeitungsstoff im 19. Jahrhundert zunahm. Dies zeigt sich schon an scheinbaren Äußerlichkeiten. Noch am Ende des 18. Jahrhunderts waren bei den deutschen Zeitungen Quartformate vorherrschend. Vermehrter Zeitungsstoff konnte allenfalls durch eine vermehrte Seitenzahl aufgefangen werden. Dern waren aber, obgleich man diesen Weg durchaus einschlug, technische Grenzen gesetzt. Die inhaltliche Erweiterung sprengte schließlich den bisher üblichen Satzspiegel. So ging man in der zweiten Hälfte des 19. Jahrhunderts zu größeren Zeitungsformaten (insbesondere Folio) über. Eine Vermehrung der Seitenzahl konnte, je nach anfallendem Stoff und den wirtschaftlichen Möglichkeiten, noch außerdem hinzukommen. Feste Werte lassen sich dafür bei der Vielzahl von Zeitungen jedoch nicht angeben.

Die Veränderungen in Format und Seitenumfang bieten nur eine äußere Annäherung an die inhaltliche Expansion der Tagespresse im 19. Jahrhundert. Ein präziseres Bild ergibt sich erst, wenn man die Erweiterung des Zeitungsstoffs genau zu quantifizieren versucht. Als Beispiel ziehe ich hierzu jetzt den "Hamburgischen Unpartheyischen Correspondenten" heran. ${ }^{20}$ Dessen Ausgaben hatten (jeweils im November) 1796 im Durchschnitt 610 Zeilen, 18561867 Zeilen und 19062731 Zeilen. Dabei ist jedoch $2 u$ bedenken, daß sich durch den Wechsel von Format und Spaltengliederung die Zeilenbreite veränderte. Berücksichtigt man

19 Schacht 1898, S. 512.

${ }^{20} \mathrm{Vgl}$. Wilke 1984, S. $97 \mathrm{ff}$. 
dies und wählt als - zugegebenermaßen sehr formales - Maß die Anzahl der Zeilenanschläge, so ergeben sich für eine Zeitungsausgabe folgende Durchschnittswerte: 179631720,185691483 und 1906138363 Anschläge.

Der Umfang des angebotenen Zeitungsstoffs pro Ausgabe stieg demnach im Laufe des 19. Jahrhunderts um mehr als das Vierfache, wobei der gröBere Teil des Wachstums schon in seiner ersten Hälfte lag. Doch muß man für ein realistisches Bild weiter in Betracht ziehen, daß der „Hamburgische Unpartheyische Correspondent” 1796 wöchentlich dreimal, 1906 hingegen 14mal (zwei Ausgaben an sieben Tagen) erschien. Rechnet man die Zeilenanschläge einheitlich auf eine Woche um, so beträgt die Steigerungsrate zwischen 1796 und 19061500 Prozent, d.h. ein Leser dieser Zeitung erhielt 1906 verglichen mit 1796 wöchentlich das Fünfzehnfache an Gedrucktem. Auch wenn der „Hamburgische Unpartheyische Correspondent" - wie schon gesagt - (schon wegen seiner häufigen Erscheinungsweise) nicht im statistischen Sinne repräsentativ für die deutschen Zeitungen steht, so gewinnt man durch ihn doch einen plastischen Eindruck, welches Ausmaß die Expansion des Zeitungsstoffs im 19. Jahrhundert annehmen konnte, ein Vorgang, den ich mit der Formel „vom Rinnsal zur Informationslawine" bezeichnet habe.

\section{III.}

Die hier zunächst in den statistischen Grunddaten beschriebene Expansion der Tagespresse im 19. Jahrhundert verlieh diesem Medium jene Omnipräsenz, wie sie uns geläufig ist. Zwar war die Presse schon am Ende des 18. Jahrhunderts das am weitesten verbreitete Druckwerk. Aber das gilt hundert Jahre später erst recht, ja in noch stärkerem Maße. Die Zeitung war zu einem allgegenwärtigen Phänomen der menschlichen Lebenswelt geworden. Dabei hatte diese Expansion politisch-rechtliche und wirtschaftliche, aber auch technische und soziale Voraussetzungen. Diese wirkten zwar letzten Endes zusammen, führten aber keineswegs zu einer linearen, sondern eher zu einer gebrochenen Entwicklung.

$\mathrm{Daß}$ nach einer kurzen Blüte im Anschluß an die Befreiungskriege die bereits erwähnten, 1819 erlassenen Karlsbader Beschlüsse wieder ein strenges Zensursystem in Deutschland einführten, ließ der Presse wenig Entfaltungs- und Bewegungsspielraum, in welcher Hinsicht auch immer. Erst die Märzrevolution 1848 brachte dieses System zu Fall. Die in den Grundrechten der Paulskirche proklamierte Pressefreiheit öffnete das Tor nicht nur für neue Zeitungsgründungen, sondern ließ auch eine andere Art von Zeitung entstehen, und zwar die Meinungs- und Parteipresse. ${ }^{21}$

$21 \mathrm{Vgl}$. Koszyk 1966, S. $127 \mathrm{ff}$. 
Doch dieser Aufschwung erlahmte schon bald, nachdem 1854 neuerlich restriktive „Bundesbestimmungen, die Verhältnisse des MiBbrauchs der Presse betreffend" ergingen. Die Gründung weiterer Presseorgane suchte man durch Konzessions- und Kautionszwang zu verhindern oder doch zu erschweren. Ein Hemmnis wirtschaftlicher Art war ferner die Stempelsteuer, die auf jede Zeitungsseite erhoben wurde. Diese bedeutete praktisch eine Kostensteigerung, die, wenn der Verleger sie auf den Leser abwälzte, zu einem höheren Bezugspreis führte und damit die Gefahr eines Auflagenverlusts in sich barg. Hinzu kam, daß der zunehmende Zeitungsstoff nicht einfach durch Vermehrung der Seitenzahl aufgefangen werden konnte. Ihn raumsparend auf möglichst wenig Seiten unterzubringen, zwang dazu, kleine Buchstabentypen zu verwenden. Dies macht viele Zeitungen der zweiten Hälfte des 19. Jahrhunderts wenig augenund leserfreundlich, was der Presseforscher noch heute „auszubaden" hat.

Die hier genannten Restriktionen wurden durch das Reichspressegesetz von 1874 aufgehoben. ${ }^{22}$ Erst jetzt wurden die Pressefreiheit gewährleistet und die Beschränkungen auf die im Gesetz selbst genannten, vergleichsweise engen Grenzen reduziert. Die damit im Rechtlichen eingetretene Liberalisierung ermöglichte eine neue Welle der Expansion im Pressewesen. Und auch der inhaltliche Spielraum der Presse erweiterte sich.

Wirtschaftliche Ursachen besaß die Expansion der Presse in der zweiten Hälfte des 19. Jahrhunderts in mehrfacher Hinsicht. Zunächst einmal hängt das Gedeihen der Presse generell mit der jeweiligen wirtschaftlichen Entwicklung zusammen. Dies gilt zumindest soweit das Pressewesen privatwirtschaftlich betrieben wird, wie es in Deutschland von Beginn an der Fall war. Zeitungen muBten primär, wenn nicht ausschließlich aus dem Bezugspreis finanziert werden, zumal es ihnen nach der Einführung des staatlichen Intelligenzmonopols Anfang des 18. Jahrhunderts untersagt war, Anzeigen (zuerst) aufzunehmen. Zum einen begrenzte der Bezugspreis die Zugänglichkeit von Presseorganen, zum anderen hing es vom wirtschaftlichen Wohlergehen ab, wie viele sich eine Zeitung leisten konnten. Unter diesen Prämissen wirkte sich der wirtschaftliche Aufschwung nach 1871 im Deutschen Kaiserreich sehr förderlich auf die Presse aus, wenn es auch schon in den siebziger und achtziger Jahren gewisse konjunkturelle Rückschläge gab.

22 Vgl. Wilke 1988 (sowie die dort verzeichnete Literatur zum Reichspressegesetz). 
Erst recht gilt dies noch in einer zweiten Hinsicht. Am 1. Januar 1850 war in Preußen das - allerdings schon in mancherlei Weise durchlöcherte - staatliche Intelligenzmonopol aufgehoben worden. Von jetzt an konnten Anzeigen ungehindert auch in die politische Tagespresse eindringen. Aus den noch bestehenden Intelligenzblättern wurden, sofern sie nicht ihr Erscheinen einstellten, im Laufe der Zeit gewöhnliche Zeitungen. Mit der Hochkonjunktur der sogenannten „Gründerzeit" gewann das Anzeigenwesen zudem eine zunehmende Marktfunktion, ja es wurde zur Verteilung der Güter und Waren geradezu unerläßlich. Verlegerische Interessen und die Interessen der Wirtschaft flossen gewissermaßen zusammen, die Zeitung wurde zu jenem „Kuppelprodukt”, von dem Karl Bücher zugespitzt gesagt hat, daf es Anzeigenraum als Ware produziert, der nur durch einen redaktionellen Teil absetzbar wird. ${ }^{23}$ Die Einnahmen aus den Anzeigen veränderten überdies die verlegerischen Kalkulationsgrundlagen. ${ }^{24}$ Und mit dem Anzeigenteil wuchs auch der redaktionelle Teil an. ${ }^{25}$

Hatten sich die Zeitungen bis zur Mitte des 19. Jahrhunderts - wie schon gesagt - im wesentlichen aus dem Vertriebserlös finanziert, so wurde in der zweiten Jahrhunderthälfte der Anzeigenerlös zur vorrangigen Finanzierungsquelle. Daraus ergab sich nicht nur - sozusagen als soziale Konsequenz -, daß der Bezugspreis gesenkt und die Zeitung erschwinglicher wurden. Vielmehr bildete sich ein typologischer Wandel im Pressewesen heraus: Der "Generalanzeiger" entstand, ein Zeitungstyp, der ganz auf der Nutzung des Anzeigenwesens fuBte. Häufig wurden die ersten Ausgaben dieser Blätter (z.B. der „Generalanzeiger der Stadt Köln", der am 29. September 1875 zum ersten Mal erschien) sogar kostenlos verteilt oder lediglich für die Zustellgebühr, und man forderte ein (geringes) Entgelt erst, als sich die Bezieher an ein solches Organ gewöhnt hatten. ${ }^{26}$ Die Generalanzeiger erzielten denn auch als erste Zeitungen wirkliche Massenauflagen, welche die sonst übliche durchschnittliche Auflage weit übertrafen.

Doch nicht nur der Anzeigenteil war für die Generalanzeiger charakteristisch. Da die Inserenten, wie man heute sagen würde, an einer möglichst großen Zahl von "Werbekontakten" interessiert waren, mußte den Zeitungen an einer möglichst großen Leserschaft liegen. Diese erreichte man

${ }^{23}$ Vgl. Bücher 1926, S. 377.

24 Vgl. Reumann 1968.

25 Vgl. dazu Meißner 1931.

26

Vgl. Dovifat 1928, Wolter 1981. 
aber nur, wenn man im Inhalt darauf verzichtete, dezidierte politische oder weltanschauliche Positionen zu vertreten. Insofern waren die Generalanzeiger der Tendenz nach unpolitisch, was nicht heißt, daß sie damit nicht mittelbar doch politische Bedeutung besaßen. Gleichwohl stellten sie einen Zeitungstyp konträr zur Parteipresse dar. Diese war überwiegend auflagenschwach und hatte - nicht zuletzt wegen mangelnder Anzeigen und fehlender Inserenten - mit wirtschaftlichen Schwierigkeiten zu kämpfen. Thre Existenz ließ sich z.T. nur durch Aktiengesellschaften begründen und aufrechterhalten, eine im 19. Jahrhundert auch im deutschen Pressewesen neue Organisationsform.

Die Expansion des Pressewesens im 19. Jahrhundert setzte auch technische Neuerungen voraus. Die von Johannes Gutenberg Mitte des 15. Jahrhunderts erfundene Drucktechnik, die auch dem Zeitungsdruck den Weg bereitete, hat sich merkwürdigerweise bis zu Beginn des 19. Jahrhunderts kaum verändert. ${ }^{27}$ Zwar gab es beim Drucken von Zeitungen Besonderheiten. Aber weil diese wegen der Aktualität der Nachrichten rasch hergestellt werden mußten, konnten größere Auflagen wie z.B. beim "Hamburgischen Unpartheyischen Correspondenten" nur durch Nutzung bzw. "Parallelschaltung” mehrerer Druckerpressen erzeugt werden. Revolutioniert wurde diese Technik erst mit der Erfindung der Schnellpresse 1811/12 durch Friedrich Koenig. Diese wurde 1814 bei der Londoner "Times" erstmals im Zeitungsdruck eingesetzt und brachte eine Beschleunigung auf 1600 Drucke pro Stunde. Die Druckleistung betrug das Vierfache, nach einer Verbesserung bald sogar das Zehnfache der herkömmlichen Pressen. Eine weitere Produktionssteigerung brachte seit den sechziger Jahren des 19. Jahrhunderts die Rotationspresse. Auch wenn diese Technik in dem überwiegend von Kleinunternehmen geprägten Presseverlagswesen in Deutschland erst allmählich vordrang, der Übergang zur Massenpresse im späten 19. Jahrhundert wäre ohne sie nicht möglich gewesen. Druckaullagen wie die $150000 \mathrm{Ex}$ emplare des „Berliner Lokalanzeigers" ließen sich nur noch im Rotationsdruck herstellen und setzten auch zunehmend eine Großbetrieblichkeit des Presseverlags - in diesem Fall das Unternehmen August Scherls voraus. $^{28}$

Schließlich ist die Expansion des Pressewesens nicht ohne Bezugnahme auf demographische und soziale Entwicklungen zu erklären. Die Bevölkerung, die auf dem Gebiet des Deutschen Reiches 1816 23,5 Millionen betragen hatte, wuchs bis zum Ende des Jahrhunderts auf mehr als das

27 Vgl. Gerhardt 1975, 1976.

Vgl. Koszyk 1966, S. 276 ff. 
Doppelte und lag im Jahre 1900 bei 56 Millionen. ${ }^{29}$ Obwohl die $\mathrm{Zu}$ wachsraten schon in der ersten Jahrhunderthälfte beträchtlich(er) waren, setzte eine soziale Umwälzung der Bevölkerung erst mit der Industrialisierung und der Verstädterung nach 1871 ein. ${ }^{30}$ Die damals entstehenden Großstädte schufen auch ein neues Publikum für die Presse. Größere politische Partizipationschancen, steigende Mobilität, der Komplexitätszuwachs der näheren und ferneren Lebenswelt bzw. deren Unübersichtlichkeit, auch die - wenngleich noch bescheidenen - Freizeitbedürfnisse - sie begründeten eine zunehmende Nachfrage nach Information und Kommunikation. Gestützt wurde diese Nachfrage durch einen (weiteren) Rückgang des Analphabetentums bzw. einen Anstieg der Lesefähigkeit, die wiederum durch die allgemeine Schulpflichtigkeit bedingt waren. ${ }^{31}$ Diese galt in Preußen zwar schon seit 1763, wurde aber erst im 19. Jahrhundert überall zur Selbstverständlichkeit. Zu den im engeren Sinne sozialen Voraussetzungen der Expansion im Pressewesen gehörte schließlich auch die bereits erwāhnte Verbilligung der Bezugspreise, die dieses Medium den breiten Schichten der Bevölkerung erst zugänglich machte.

IV.

Indem der Umfang der Zeitungen im 19. Jahrhundert stark ausgeweitet wurde, wandelte sich auch deren Inhalt. Aufs ganze gesehen wurde dieser vielseitiger, ja es bildete sich erst vollends jene thematische Universalität heraus, die nach unseren heutigen Begriffen für die Massenmedien konstitutiv ist. Am Beispiel des ${ }_{n}$ Hamburgischen Unpartheyischen Correspondenten", aber auch an anderen Organen, ist ablesbar, daB am Ende des 18. Jahrhunderts noch die politisch-militärische Berichterstattung dominierte. ${ }^{32}$ Bis zum Ende des 19. Jahrhunderts hatte sich dann der Zeitungsinhalt diversifiziert. Vermehrt fanden Berichte über Handel und Wirtschaft, über Recht und Soziales sowie über Kultur in den Zeitungen Aufnahme, obwohl die Anfänge eines „Kulturteils" beim „Hamburgischen Unpartheyischen Correspondenten” schon im 18. Jahrhundert lagen. Das Wirklichkeitsspektrum, über das berichtet wurde, dehnte sich aus, immer mehr Dinge wurden auch zum Gegenstand der "Medienrealität", wie wir das in den Medien vermittelte Bild der Welt inzwischen nennen.

29 Vgl. Marschalk 1984.

$30 \mathrm{Vgl}$. Reulecke 1985.

31 Vgl. Engelsing 1973, S. $96 \mathrm{ff}$.

32 Vgl. Wilke 1984, S. 124 ff. 
Verändert hat sich in den Zeitungen des 19. Jahrhunderts auch die geographisch-politische Herkunft und Zusammensetzung der Berichterstattung. Wieder zeigt das Beispiel des „Hamburgischen Unpartheyischen Correspondenten" bis zum Ende des 18. Jahrhunderts eine Dominanz der Information aus dem Ausland, d.h. von jenseits der Grenzen des Deutschen Reiches. ${ }^{33}$ Zu Beginn des 20. Jahrhunderts steliten „nationale" und „internationale" Nachrichten dann einen etwa gleich großen Anteil. Doch war außerdem etwas hinzugekommen, was es im 18. Jahrhundert - zumindest in den politischen Zeitungen - noch kaum gegeben hatte, nämlich die lokale Berichterstattung. Ansätze hierzu waren zwar in den Intelligenzblättern vorhanden, aber ein eigener Lokalteil entstand erst im (späteren) 19. Jahrhundert. ${ }^{34}$ In doppelter Weise nämlich "national" und „lokal” - gewann der Zeitungsinhalt damals wesentlich an Nähe zum Publikum.

Zum Funktionswandel der Presse trug im 19. Jahrhundert noch etwas anderes bei. Bis zum Ende des 18. Jahrhunderts war in Deutschland der Typ der referierenden, „relatorischen" Zeitung vorherrschend. Das Etikett „unparteïsch" im 'Titel des „Hamburgischen Unpartheyischen Correspondenten" kann dafür als symptomatisch gelten. Bedingt war diese Haltung nicht nur durch die Zensur, sondern sie entsprach auch dem journalistischen Selbstverständnis, wonach man den Leser zu unterrichten habe, ihm seine Meinungsbildung aber selbst überlasse. Mit dem im späten 18. Jahrhundert auch in Deutschland einsetzenden Kampf um Pressefreiheit drängten die Journalisten dann darauf, in den Zeitungen auch ihre Meinung zu äußern und Kritik zu üben. Wo solche Pressefreiheit im frühen 19. Jahrhundert (zeitweise) gewährt wurde, bildeten sich die Anfange einer meinungsbetonten Presse heraus. Als exemplarisch wird dafür immer wieder Joseph Görres' "Rheinischer Merkur” von 1814/16 angeführt. Auf breiter Front brach das Zeitalter der Meinungspresse aber erst nach 1848 an. Mit den sich im Parlamentarismus formierenden Parteien entstanden auch Parteizeitungen, die die jeweiligen politischen und weltanschaulichen Positionen öffentlich vertreten sollten. Aber selbst wenn solche Organe nicht unmittelbar einer Partei zugehörten oder ihr nahestanden, so waren sie doch häufig richtungsbestimmt und machten das Pressewesen tendenzhaltig. ${ }^{35}$ Auf der anderen Seite verringerten sich die Absatzchancen im Prinzip um so mehr, je

33 Wilke 1984, S. $147 \mathrm{ff}$.

34 Vgl. Conrad 1935.

$35 \mathrm{Vgl}$. Die Gruppierung der Zeitungen nach ihrer Parteistellung bei Schacht 1898 , S. 518, vgl. auch Roth 1913. 
enger die Richtung jeweils festgelegt war; weshalb - wie schon erwähnt - im späten 19. Jahrhundert die meinungslosen Generalanzeiger in der Regel die erfolgreicheren Blätter waren.

Noch in anderer Hinsicht wandelten sich Inhalt und Funktion der Presse im 19. Jahrhundert, und zwar insonderheit in den Jahrzehnten nach 1870. Seitdem nahmen die Zeitungen vermehrt auch phantasiebetonte, erzählende, wenn man so will „literarische" Formen und Beiträge auf. ${ }^{36}$ Gemeint sind damit der Zeitungsroman, Reiseskizzen, Kurzgeschichten, das Feuilleton als "kleine Form". Damit übernahm die Tagespresse für ihre Leser auch die Aufgabe der "Unterhaltung”. Nachdem der Stuttgarter Verlagsbuchhändler und Druckereibesitzer Hermann Schönlein 1872 damit begonnen hatte, führten die politischen Zeitungen großenteils sogar eigene Unterhaltungsbeilagen ein, deren Stoff z.T. eigene Feuilletonkorrespondenzen lieferten. (Übrigens gab es Beilagen auch zu anderen Lebensbereichen, zur Gesundheitspfiege, Rechtskunde u.ä.). Wenn heute solch unterhaltende Beiträge aus den Tageszeitungen fast ganz verschwunden sind und allenfalls noch in der Wochenendausgabe vorkommen, so dürfte dies seinen Grund darin haben, daß die Funktion, die sie einst erfüllten, inzwischen von anderen Medien - insbesondere dem Fernsehen - wahrgenommen wird, und dies bestimmt auch noch besser.

\section{V.}

Es liegt auf der Hand, daß die beschriebenen Verãnderungen der Presse auf dem Wege zur "Großmacht" im 19. Jahrhundert auch ihre sprachlichen Aspekte hatten. Dies kann hier naturgemäß nicht im einzelnen dargestellt, sondern nur angedeutet werden. Es müBte zudem auch erst noch untersucht werden, womit ich an dieser Stelle freilich Eulen nach Athen trage. Zunächst einmal bedeutete die zuvor geschilderte Expansion, daß die Tagespresse im 19. Jahrhundert einen wachsenden, zu beträchtlichem Ausmaß ansteigenden Beitrag zur gesellschaftlichen Sprachverarbeitung leistete. Wenn die alltägliche Sprachproduktion und Sprachverarbeitung damals stark zugenommen haben, so hatte die Presse daran erheblichen Anteil. Genau quantifizieren läßt sich dieser Anteil nicht, wohl aber, wie wir gesehen haben, die Sprachvermehrung in den Zeitungen selbst. Das Auszählen der Zeilenanschläge gab einen Eindruck davon.

Sprachlich von Bedeutung war aber nicht nur die Expansion im ganzen, sondern auch Veränderungen im einzelnen. Beispielsweise läßt sich eine Zunahme im durchschnittlichen Umfang der Zeitungsbeiträge feststel-

36 Vgl. zum Beleg Wilke 1984, S. $108 \mathrm{ff}$., dazu auch Stoklossa 1910. 
len. ${ }^{37}$ In der Frühzeit der Presse hatten die Nachrichten in der Mehrzahl der Fälle nur aus (allenfalls) einem Satz bestanden und blieben in ihrem Informationsgehalt folglich weitgehend am äußeren, vordergründigen Geschehen hängen. Dies hatte sich zwar schon im Laufe des 18. Jahrhunderts zu ändern begonnen, der Wandel schritt aber im 19. Jahrhundert weiter voran. Im Jahre 1906 bestanden nur noch 27 Prozent der Nachrichten im „Hamburgischen Correspondenten” aus einem Satz. Man kann folglich sagen, daß der Informationsgehalt der Presse im 19. Jahrhundert auch im einzelnen (weiter) zunahm, d.h. der Komplexitätsgrad der mitgeteilten Informationen wurde größer. Und damit kamen zwangsläufig auch komplexere sprachliche Strukturen bei der journalistischen Textbildung zustande.

Die Rolle der Presse für die Sprache läßt sich - vereinfacht gesehen - in zweifacher Weise bestimmen. Zum einen ist sie ein Multiplikator von Sprachformen, die unabhängig von ihr im Gebrauch sind. Insofern dringen im 10. Jahrhundert mit der Expansion der Presse auch gewisse Sprachbestände (vor allem lexikalische) in eine breitere Öffentlichkeit vor. Zum anderen bildet die Presse aufgrund ihrer Funktionen selbst bestimmte Sprachformen aus. $\mathrm{Ob}$ man damals schon von einer eigenen "Zeitungssprache" sprechen kann, wie man es für später getan hat, muß hier dahingestellt bleiben. $\mathrm{Zu}$ den Stilformen, die durch die Presse im späten 19. Jahrhundert geschaffen werden, gehört jedenfalls die Schlagzeile. $^{38}$ In der frühen Presse wurden Nachrichten zunächst nur unter dem Datum und Namen des Herkunftorts zusammengefaßt. Im 18. Jahrhundert findet man am ehesten allenfalls das Feuilleton unter einer eigenen Überschrift ausgesondert ("Von merkwürdigen und gelehrten Sachen"). Doch erst als die Zeitungen im 19. Jahrhundert in Umfang und Inhalt stark zulegten, wurden Überschriften unerläBlich, und zwar - zusammen mit drucktechnischen Formen der Gliederung und "Aufmachung" - als Selektionshilfe für den Leser, der gar nicht mehr alles in der Zeitung lesen konnte. ${ }^{39}$ Dabei handelte es sich z.T. noch um bloße Ressortbezeichnungen oder um die Etikettierung der Nachrichtenart (etwa "Letzte Telegramme"). Wo aber bereits Themaüberschriften auftraten, geschah dies häufig schon in der Form von Nominalsätzen, wie wir sie auch heute noch von Schlagzeilen kennen. Gleichwohl ist die leserwerbende Funktion der Schlagzeile in Deutschland erst im 20. Jahrhundert voll durchgedrungen.

37 Vgl. Wilke 1984, S. $100 \mathrm{f}$.

$38 \mathrm{Vgl}$. Sandig 1971, S. $132 \mathrm{ff}$.

30 Vgl. Wilke 1984, S. $101 \mathrm{ff}$. 
Bisher war ausschließlich von Zeitungen die Rede, obwohl zur Presse auch die Zeitschrift als publizistische Gattung gehört. Auf deren Entwicklung im 19. Jahrhundert kann hier nicht in ähnlicher Weise eingegangen werden. ${ }^{40}$ Doch sei zumindest darauf hingewiesen, daß in dieser Zeit zugleich auch eine Expansion im Zeitschriftensektor stattfand. Zeitschriften, das sind in mehr oder weniger großen Intervallen erscheinende Druckwerke, die einem umgrenzten Aufgabenbereich und damit häufig zugleich einer bestimmten Zielgruppe von Lesern dienen. Solche Zeitschriften waren in großer Zahl schon im 18. Jahrhundert herausgekommen und sie hatten sich dabei typologisch und thematisch differenziert. Die bereits damals immer wieder beklagte Flut von Titeln bewirkte, daß viele keine längere Erscheinungsdauer zu erreichen vermochten.

Im 19. Jahrhundert, zumal in seinen letzten Jahrzehnten, wuchs auch die Zahl der Zeitschriften weiter an. Lag ihre Zahl in der ersten Jahrhunderthälfte in Deutschland noch unter 500, so waren es um $1900 \mathrm{ca}$. $6.000 .{ }^{41} \mathrm{Zu}$ dieser Expansion war es vor allem seit den siebziger Jahren gekommen. Allein in den neunziger Jahren wurde die Gründung von 1704 Zeitschriften registriert. Dabei handelte es sich mehrheitlich um Fachzeitschriften. Die fachliche und soziale Differenzierung der Gesellschaft bildete den entscheidenden Antriebsfaktor für die Spezialisierung des Zeitschriftenwesens. Doch erschienen neben der Vielzahl von Fachzeitschriften mit ihren überwiegend kleinen Auflagen in der zweiten Hälfte des 19. Jahrhunderts eine ganze Reihe von Publikumszeitschriften, die sich an eine breite Leserschaft wandten. Hierzu gehörten vor allem $\mathrm{Fa}$ milienblätter wie „Die Gartenlaube" (1853 ff.) und „Daheim” (1864 ff.). Die Leipziger "Illustrirte Zeitung" war zwar schon 1843 gegründet worden, sollte jedoch ihre Blüte erst später erleben wie die 1891 erstmals erschienene "Berliner Illustrirte Zeitung". Publizistische Neuerungen wie diese entstammen somit ebenfalls (noch) dem 19. Jahrhundert und waren Bestandteil des expansiven Pressewesens.

\section{VII.}

Wenn es zutrifft, daß die Presse im 19. Jahrhundert zu einer ${ }_{n}$ Großmacht" wurde, so muß damit zugleich eine Machtverschiebung im Verhältnis zu den angestammten Gewalten und gesellschaftlichen Machtgebilden eingetreten sein. War die Presse diesen einst untergeordnet, so

40 Zum Überblick vgl. Kirchner 1962.

41 Vgl. Kootz 1908. 
erhob sie jetzt zunehmend den Anspruch, gleichrangig zu sein, ja diese in ihrer Geltung zu relativieren. Der eingangs zitierte Domkapitular Molitor sprach davon, die Presse sei zu einem Hauptmittel geworden, „um Throne zu stürzen, um den Altar zu zertrümmern";2 und er sprach damit metaphorisch Staat und Kirche als die beiden Institutionen an, die offenbar in erster Linie vom Machtzuwachs der Presse in Mitleidenschaft gezogen wurden.

Eine Ahnung davon hatte in den vierziger Jahren des 19. Jahrhunderts schon der Staatsrechtler Robert von Mohl besessen, als er schrieb, „daß das Regieren durch die in dem Geiste der Zeitungen in allen gesittigten Staaten vorangegangene Veränderung sehr bedeutend schwüriger geworden ist, und daB also itzt mehr Kraft und Klugheit dazu gehört, als früher." ${ }^{43}$ Gut zwei Jahrzehnte später hatte sich die Situation in den Augen mancher Beobachter so verschärft, dafi sie zu Worten wie den folgenden griffen: "So viel ist gewiß, die moderne Presse und der moderne Staat ringen einen Kampf auf Leben und Tod: eines von beiden muB unterliegen. Die Mitregentschaft, welche der Staat anbietet, erträgt die Presse nicht. Sie weib nichts von Brüderschaft und zieht, wie man im gemeinen Leben zu sagen pflegt, die ganze Decke an sich." 44 Sicher entstammen solche Worte einem noch stark obrigkeitlichen Staatsverständnis und einem fehlenden Begriff von Demokratie. Aber sie signalisieren doch deutlich die unzweifelhaft empfundene Machtverschiebung.

Wie reagierte der Staat auf diese Machtverschiebung? Nachdem ihm 1848 das Mittel der Zensur entzogen und mit dem Erlaß des Reichspressegesetzes 1874 die verbliebenen Instrumente der Reglementierung genommen worden waren, wurden im wesentlichen zwei Wege beschritten. Einerseits verlagerte sich die Auseinandersetzung vor Gericht. Vor allem Otto von Bismarck verfolgte als preuBischer Ministerpräsident und später als Reichskanzler ihm nicht genehme Blätter und Journalisten häufig mit Anklagen. Andererseits ging man dazu über, eine aktive Pressepolitik zu betreiben, bei der man sich wiederum verschiedener Mittel bediente. ${ }^{46}$ Zu den weniger legitimen gehörte z.B. die Bezahlung oder gar Bestechung von Journalisten. Im Kern der amtlichen Pressepolitik ging es um die inhaltliche Einflußnahme auf die Presse. Dazu nutzte Bismarck u.a. die Provinzialkorrespondenz, die die Kreisblätter mit gouvernemental in-

42 Molitor 1866, S. 7.

43 Vgl. von Mohl 1845, S. 128 Anm. 3.

44

Vgl. Fischer-Frauendienst 1963, Naujoks 1967. 
spirierten Artikeln versorgen sollte. Nach der Reichsgründung setzte der Aufbau eines amtlichen Pressereferats im Auswärtigen Amt ein. Dieses gewann allerdings nur allmählich Einfluß und bot lange Zeit AnlaB, über die mangelnde Koordinierung der amtlichen Informationspolitik zu klagen. Schon seit den sechziger Jahren hatte zudem das "Wolffsche Telegraphen-Büro", die erste deutsche Nachrichtenagentur, eine halbamtliche, offiziöse Stellung. All diese Mittel zielten darauf ab, den Vorrang der politischen Herrschaft vor der "Mitregentschaft" durch die Presse zu wahren.

Unter den staatlichen Gewalten sah man übrigens auch die Justiz beeinträchtigt. So äußerte der bereits zitierte Joseph Lukas: „Die Macht der Presse ist bereits so groB geworden, daB sie selbst den Richterstand mitunter terrorisirt. Bei den Geschwornen-Gerichten ist das eingestandenermaßen der Fall; in sogenannten berühmten Fällen schöpfen die Geschwornen ihr Urteil aus der 'öffentlichen Meinung', nicht aber aus ihrer inneren Überzeugung." 46 Die Unabhängigkeit der juristischen Urteilsfindung schien angeblich nicht mehr gewährleistet, sondern durch die Rücksichtnahme auf die „öffentliche Meinung” gefährdet. Und diese wird hier wiederum als ein Produkt der Presse gesehen.

$\mathrm{Daß}$ mit Hilfe der Presse Altäre zertrümmert würden, mußte die Kirche als andere, mit Macht ausgestattete Institution herausfordern. Von kirchlichen Kreisen ging ja auch die Debatte über die zur "GroBmacht" gewordene Presse nicht zuletzt aus. Die Kritik entzündete sich hier vor allem an zwei Dingen: Einerseits am Vordringen der Presse des politischen Liberalismus, der man antiklerikale Einseitigkeit und tendenziöse Färbung vorwarf. Man sah sie gewissermaßen auf der Woge des Zeitgeistes schwimmen, und diesem muBte Widerstand geleistet werden. Andererseits nahm man Anstoß daran, daß die Presse zu einem Industriezweig geworden, ja zur „reinen Geschäftssache" ${ }^{17}$ herabgesunken sei. Darin erblickten nicht wenige die entscheidende Ursache einer fatalen Entwicklung, die dazu zwang, nach dem Publikum zu schielen und dieses mit Sensationen zufriedenzustellen. Später richtete sich die Kritik aber nicht weniger auch gegen die sogenannte "farblose Presse". Diese unterlasse zwar offene Angriffe auf die Kirche, verbreite unter dem Deckmantel der Neutralität aber die pure Grundsatzlosigkeit. Der Bedrohung von beiden Seiten mußte die Kirche indessen anders begegnen als der Staat. Auf ihrer Seite folgte aus der Diagnose unmittelbar die Therapie, der "schlechten"

46 Lukas 1867, S. 185.

47 Lukas 1867 , S. 45. 
Presse praktisch eine ${ }_{\text {gute}}$ "Presse entgegenzusetzen ${ }^{48}$ denn, so fragte

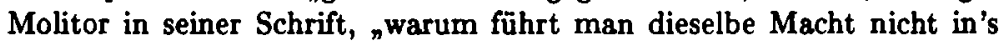
Feld für die Wahrheit?"40

Von der Presse als einer "Großmacht", ja einer „Allmacht"50 zu sprechen, schließt die Annahme großer Medienwirkungen ein. Diese Annahme mag in manchen Formulierungen der zitierten Quellen kulturpessimistisch überzogen erscheinen. Sie ist jedoch im späten 19. Jahrhundert als solche ein soziales Phänomen, auch wenn es seinerzeit noch an den Mitteln fehlte, einen wissenschaftlichen Nachweis dafür zu führen. Als dies seit den dreißiger Jahren des 20. Jahrhunderts empirisch möglich wurde, wurde die These von den großen Medienwirkungen zunächst revidiert. Doch hat sich inzwischen gezeigt, daß dies nur bedingt richtig war und daß es doch hinreichend Gründe gibt, große Wirkungen der Massenmedien anzunehmen, wenn auch vor allem beim noch vergleichsweise jungen Fernsehen. ${ }^{51}$

Die Macht der Presse hat man schon im 19. Jahrhundert vornehmlich darin gesehen, daß sie die Realität nicht objektiv wiedergibt, sondern subjektiv gefiltert. Diesen Vorgang findet man in ein Bild gekleidet, das recht aktuell anmutet. Die Presse fungiere nicht als ein "Planspiegel", sondern als "verzerrender Hohlspiegel", heißt es bei einem der Zeitgenossen. ${ }^{52}$ Außer verzerrten Vorstellungen im Bewußtsein der Bevölkerung wurden auch bereits Wirkungen auf die (politische) Realität selbst beobachtet. Heinrich Wuttke meinte jedenfalls, dergleichen im Zusammenhang der preußisch-österreichischen Auseinandersetzungen von 1866 feststellen zu können, die bekanntlich zur "kleindeutschen" Lösung führten. Gerade sprachliche Strategien machte er dafür verantwortlich. Die "ungeheuere Gewalt der Zeitungen" bestehe darin, "das Urtheil der allermeisten Menschen gefangen zu nehmen und ihre gesunde Vernunft zu verwirren, indem sie Worte, die an sich deutlich und sprechend einen unbestrittenen Sinn haben, veränderte selbst entgegengesetzte Bedeutung unterzuschieben vermochten". ${ }^{53}$ Und Wuttke war es auch, der

48 Vgl. Schmolke 1971.

49 Molitor 1866, S. 7.

50 Zöckler 1870, S. 7.

51 Als Überblick zur heutigen Wirkungsforschung vgl. Schenk 1987, NoelleNeumann 1989.

52 Zöckler 1870, S. 19.

${ }^{53}$ Wuttke 1875, S. 196. 
erkannte, då die eigentlichen Konsequenzen der Medienkommunikation langfristiger Natur sind. Hellsichtig konstatierte er 1875: „Die Zeitungen selbst sind bald in Vergessenheit verfallen, jedoch die Wirkungen, welche sie während ihrer Herrschaft zu Wege gebracht haben, dauern weiter bestimmend fort." $\$ 4$

\section{Literatur}

Bogel, Else/Blühm, Elger (Hrsg.) (1971, 1985): Die deutschen Zeitungen des 17. Jahrhunderts. Ein Bestandsverzeichnis mit historischen und bibliographischen Angaben. 2 Bde. Bremen 1971. 3. Nachtragsband München 1985.

Bücher, Karl (1926): Gesammelte Aufsätze zur Zeitungskunde. Tübingen.

Conrad, Erich (1935): Die Entwicklung des lokalen Teils der größeren Leipziger Tageszeitungen in der 2. Hälfte des 19. Jahrhunderts. Diss. Leipzig.

Cooke, Jacob E. (1961): The Federalist. Middletown.

Dovifat, Emil (1928): Die Anfänge der Generalanzeigerpresse. In: Archiv für Buchgewerbe und Gebrauchsgraphik 65, S. 163-184.

Engelsing, Rolf (1971): Analphabetentum und Lektüre. Zur Sozialgeschichte des Lesens in Deutschland zwischen feudaler und industrieller Gesellschaft. Stuttgart.

Erler, Curt (1911): Von der Macht der Presse in Deutschland. Berlin.

Fischer-Frauendienst, Irene (1963): Bismarcks Pressepolitik. Münster.

Gerhardt, Claus W. (1975): Geschichte der Druckverfahren. Teil II: Der Buchdruck. Stuttgart.

Gerhardt, Claus W. (1976): Warum wurde die Gutenberg-Presse erst nach über 350 Jahren durch ein besseres System abgelöst? In: Gerhardt, Claus W.: Beiträge zur Technikgeschichte des Buchwesens. Kleine Schriften 19691976. Frankfurt/M., S. 79-100.

Hammer, Philipp (1868): Die Presse, eine Großmacht, oder ein Stück moderner Versimpelung? Würzburg.

von Hassell, Ulrich (1902): Deutsche Zeitschriften und ihre Wirkung auf das Volk. Stuttgart.

54 Wuttke 1875 , S. 218. 
Heenemann, Horst (1929): Die Auflagenhöhe der deutschen Zeitungen. Ihre Entwicklung und ihre Probleme. Diss. Berlin.

Hoefer, Frank Thomas (1983): Pressepolitik und Polizeistaat Metternichs. Die Überwachung von Presse und politischer Öffentlichkeit durch das Mainzer Informationsbüro (1833-1848). München/New York/London/Paris.

Kirchner, Joachim (1962): Das deutsche Zeitschriftenwesen. Seine Geschichte und seine Probleme. Teil II: Vom Wiener Kongreß bis zum Ausgang des 19. Jahrhunderts. Wiesbaden.

Kootz, Robert (1908): Zur Statistik der deutschen Zeitschriften. In: Zeitschrift für die gesamte Staatswissenschaft 64, S. 526-560.

Koszyk, Kurt (1966): Deutsche Presse im 19. Jahrhundert. Berlin.

La statistique des journaux (1908): In: Bulletin de l'Institut International de Statistique 17, S. $176 \mathrm{ff}$.

Lukas, Joseph (1867): Die Presse, ein Stück moderner Versimpelung. Regensburg/New York/Cincinnati.

Marschalk, Peter (1984): Bevölkerungsgeschichte Deutschlands im 19. und 20. Jahrhundert. Frankfurt/M.

Meißner, Hans-Heinz (1931): Das Inserat in den groben deutschen politischen Tageszeitungen von 1850 bis 1870 . Diss. Leipzig.

von Mobl, Robert (1845): Die Polizei-Wissenschaft nach den Grundsätzen des Rechtsstaates. Bd. 3: System der Präventiv-Justiz oder Rechts-Polizei. 2. Aufl. Tübingen.

Molitor, Wilhelm (1866): Die Großmacht der Presse. Ein Wort für unsere Tage aus der mitteleuropäischen Staatengruppe. Regensburg/New York.

Muser, Gerhard (1918): Statistische Untersuchung über die Zeitungen Deutschlands 1885-1914. Diss. Leipzig.

Naujoks, Eberhard (1967): Bismarck und die Regierungspresse. In: Historische Zeitschrift 20, S. 46-80.

Noelle-Neumann, Elisabeth (1989): Wirkung der Massenmedien. In: NoelleNeumann, Elisabeth/Schula, Winfried/Wilke, Jürgen (Hrsg.): Fischer Lexikon Publizistik/Massenkommunikation. Frankfurt/M., S. 360-400. 
von Reden (1848): Statistische Ergebnisse der deutschen periodischen Presse. In: Zeitschrift des Vereins für deutsche Statistik 2, S. 244-250.

Reulecke, Jürgen (1985): Geschichte der Urbanisierung in Deutschland. Frankfurt/M.

Reumann, Kurt (1968): Entwicklung der Vertriebs- und Anzeigenerlöse im Zeitungsgewerbe seit dem 19. Jahrhundert. In: Publizistik 13, S. 226-271.

Ronneberger, Franz (1983): Publizistische und politische Macht. In: Rundfunk und Fernsehen 31, S. 260-270.

Roth, Paul (1913): Die Programme der politischen Parteien und die politische Tagespresse in Deutschland. Halle.

Sandig, Barbara (1971): Syntaktische Typologie der Schlagzeile. Möglichkeiten und Grenzen der Sprachökonomie im Zeitungsdeutsch. München.

Schacht, Hjalmar (1898): Statistische Untersuchung über die Presse Deutschlands. In: Jahrbücher für Nationalökonomie und Statistik, III. Folge. Bd. 15. Jena, S. 503-525.

Schenk, Michael (1987): Wirkungsforschung. Tübingen.

Schmolke, Michael (1971): Die schlechte Presse. Katholiken und Publizistik zwischen "Katholik" und "Publik" 1821-1968. Münster.

Stoklossa, Paul (1910): Der Inhalt der Zeitung. In: Zeitschrift für die gesamte Staatswissenschaft 26, S. 555-565.

Stoklossa, Paul (1913): Die periodischen Druckschriften Deutschlands. Eine statistische Untersuchung. In: Schmollers Jahrbuch für Gesetzgebung, Verwaltung und Volkswirtschaft im Deutschen Reich 37, S. 225-258.

Welke, Martin (1977): Zeitung und Öffentlichkeit im 18. Jahrhundert. Betrachtungen zur Reichweite und Funktion der periodischen deutschen Tagespublizistik. In: Presse und Geschichte. Beiträge zur historischen Kommunikationsforschung. München, S. 71-99.

Wilke, Jürgen (1984): Nachrichtenauswahl und Medienrealität in vier Jahrhunderten. Eine Modellstudie zur Verbindung von historischer und empirischer Publizistikwissenschaft. Berlin/New York.

Wilke, Jürgen (1985): Mainzer Lesegesellschaften. In: Buchhandelsgeschichte H. 1, S. B1-B9. 
Wilke, Jürgen (1988): Die periodische Presse im Kaiserreich. In: Archiv für Geschichte des Buchwesens 31, S. 221-230.

Wilson, Francis G. (1942): "The Federalist" on Public Opinion. In: Public Opinion Quarterly 6, S. 563-575.

Wolter, Hans-Wolfgang (1981): Generalanzeiger - Das pragmatische Prinzip. Bochum.

Wuttke, Heinrich (1875): Die deutschen Zeitschriften und die Entstehung der öffentlichen Meinung. Ein Beitrag zur Geschichte des Zeitungswesens. 2. Auf. Leipzig.

Zöckler, Otto (1870): Der Einfluß der Presse auf das menschliche Leben der Gegenwart. Berlin. 